\title{
RADIO SAGO: ENTRE EL IDEARIO REGIONALISTA Y EL APOYO \\ A LA DICTADURA
}

\author{
OSCAR CONTRERAS FUENTEALBA ${ }^{1}$
}

Fecha de recepción: febrero de 2020

Fecha de aceptación y versión definitiva: octubre de 2020

\begin{abstract}
RESUMEN: El presente artículo busca remarcar el mensaje demagógico y antimarxista que puso en circulación Alfonso Sanhueza (secretario general de la institución por 35 años) a través de Radio SAGO. La emisora se transformó en un dispositivo divulgador de la clase terrateniente local. Estos argumentos fueron utilizados como elementos aglutinadores, sobre todo el ámbito del agro, para crear una conciencia regional que acercara los intereses de los terratenientes al común de los ciudadanos.
\end{abstract}

PALABRAS CLAVE: Radio SAGO; conciencia regional; manipulación de la información; marxismo; dictadura militar.

\section{Radio Sago: Between the Regionalist Idea and Support for the Dictatorship}

ABstRACT: This article seeks to highlight the demagogic and anti-Marxist message that Alfonso Sanhueza (secretary general of the institution for 35 years) put into circulation through Radio SAGO. The station was transformed into a local landowner class-disseminating device. These arguments were used as binding elements, especially in the field of agro, to create a regional awareness that would bring the interests of landowners closer to the common of citizens.

KEY WORDS: Radio SAGO; regional awareness; information manipulation; Marxism; military dictatorship.

\footnotetext{
1 Profesor de Historia y Geografía y Magíster en Ciencias Humanas, mención Historia, titulado en Universidad de Los Lagos, Chile. Correo electrónico: oscar.contreras.fuentealba@gmail.com.
} 


\section{INTRODUCCIÓN}

Creada el 4 de septiembre de 1939, la emisora de la Sociedad Agrícola y Ganadera de Osorno (SAGO), conocida como Radio SAGO, comenzó dando avisos, adelantos agropecuarios y programas radiales. Durante 1973 a 1980 hace notar, desde antes del Golpe de Estado, su pertenencia a los grupos más acomodados de la región, siendo contraria a cualquier tipo de reforma: «La colectivización de la tierra conduce a la peor de las esclavitudes porque transforma al hombre en siervo del Estado, llegando a negarle hasta el uso del fruto de su trabajo y despojándolo de todo aquello que sublimiza su labor» (Sanhueza, 1973).

En la antesala del derrocamiento de Salvador Allende, la Radio SAGO no sólo se levantó como un bastión de resistencia burguesa a nivel productivo, sino que, con una estrategia comunicacional que se mantuvo durante toda la Dictadura, apeló a la moral, fundamentalmente a los valores cristianos y a la familia, para sustentar al nuevo gobierno. Alfonso Sanhueza (1973), en su boletín diario sacó a relucir lo que, según él, era una ausencia de valores que afectaba al país, y que en su opinión era responsabilidad de los partidos de izquierda:

Su propósito es, según lo que gritan a todo gaznate, tomarse la calle, y en seguida proceder a aniquilar la vida hogareña desatando el terror [...], así se contribuye a la destrucción del régimen democrático, de la libertad de trabajo, de la libre expresión de las ideas y de la convivencia familiar dentro de nuestros hogares.

Además, en conjunto con el antimarxismo, la SAGO puso en circulación un ideario regionalista centrado en los intereses agropecuarios de los grandes terratenientes. Siguiendo los postulados de Foucault (1977), esta emisora se transformó en un dispositivo de divulgación, producto de su función estratégica dominante, moldeando, controlando e influyendo conductualmente.

Al mismo tiempo construía la nueva imagen gubernamental, cimentándola en la figura de Diego Portales ${ }^{2}$. Lejos de profundizar en los aspectos técnicos de su cometido, lo utilizaban como un modelo de orden y autoritarismo.

2 Fue un político y comerciante perteneciente a la oligarquía chilena. Ocupó cargos ministeriales desde1830 a su muerte en 1837. Se le atribuye ser el principal ideólogo en la Constitución de 1833, y haber consolidado una estabilidad política a través de medidas autoritarias. De acuerdo con Jocelyn Holt (1998): «Para Portales [...] lo que realmente importaba era lo que él denominó «el peso de la noche», esto es, la sumisión social de las clases populares, el orden señorial y jerárquico que verdaderamente presidía y gobernaba el país» (p. 27). 


\section{METODOLOGÍA}

La investigación presentada se enmarca en la línea cualitativa. El estudio de la Radio SAGO responde a un contexto de censura, control y un fuerte roce entre sectores de la población, y enmarcarnos en este tipo de metodología permitió abordar la temática de manera de analizar los aspectos más relevantes del discurso:

Los de orden cualitativo se centran en la comprensión de una realidad considerada desde sus aspectos particulares como fruto de un proceso histórico de las construcciones y vista a partir de la lógica y el sentir de sus protagonistas, es decir, desde una perspectiva interna (Pérez, 2001, p. 48).

Bajo este mismo punto de vista, nos es esencial para comprender los significados y definiciones, considerando el valor ideológico en su contenido (Salgado, 2007), buscando codificar el mensaje dentro de la complejidad del objeto de estudio, entendiendo que es un conglomerado de elementos únicos, intentando reconstruir su postura, dando a conocer los temas más desarrollados (Canales, 2006). Como argumenta Martínez (2006), «la investigación cualitativa trata de identificar la naturaleza profunda de las realidades, su estructura dinámica, aquella que da razón plena de su comportamiento y manifestación» (p. 128).

En vista de aquello, el objetivo del presente artículo es analizar el mensaje de Radio SAGO durante el periodo dictatorial (1973-1980) a través del Fondo Sanhueza ${ }^{3}$. Considerando como coyunturas el Golpe de Estado de 1973 y la instauración de la Constitución de 1980. Por lo tanto, la pregunta guía es la siguiente: ¿Cuál es la lógica sobre la que operaban los dispositivos de poder a la hora de divulgar su mensaje radial?

A la vez complementamos nuestro enfoque con la metodología utilizada por Kristin Sorensen (2015) en Los medios, la memoria y los derechos humanos en Chile. Nos resulta útil ya que aplica un modelo interdisciplinario que considera el análisis de los textos de los medios de comunicación, además del contexto cultural en que se emite el mensaje, evaluando su influencia en el entorno. Como indica la investigadora estadounidense: «Los medios de comunicación y su cultura circundante desempeñan un papel clave en la formación de identidades y conciencias locales, nacionales y mundiales» (p. 45).

3 La donación fue realizada por los hijos de Alfonso Sanhueza el año 2008 a Universidad de Los Lagos. La cronología de estos documentos va desde 1949 a 1990. El editorial se emitía diariamente por Radio SAGO, tratándose temas de agricultura, política e interés regional. Actualmente la colección está digitalizada y abierta a quien la solicite. 
Como fuente histórica central son tomados los boletines editoriales emitidos desde 1973 a 1980 a las 13 horas en Radio SAGO (Fondo Sanhueza). Documentos redactados por Alfonso Sanhueza. Estos archivos se encuentran en dependencia del Programa de Estudios y Documentación en Ciencias Humanas (PEDCH) de la Universidad de Los Lagos (Osorno, Chile). Es importante recalcar que es una fuente inédita. No se tiene registros de investigaciones que consideraran dicha fuente como objeto de estudio central. También se han consultado algunos artículos extraídos desde el diario La Prensa.

\section{HIPÓTESIS}

Radio SAGO enalteció los símbolos patrios y a las FF.AA. con la finalidad de robustecer un conglomerado militar que recientemente había arrebatado el poder a los socialistas. No obstante, el planteamiento principal va más allá de un nacionalismo clásico. Este medio de comunicación local centró su foco de atención en crear un sentimiento regionalista y localista en Osorno y alrededores. Bajo esta lógica, la SAGO fue el organismo más activo en utilizar la temática agrícola como elemento aglutinador. La emisora intentó moldear y dar forma a la identidad local, camuflando los intereses de los grandes terratenientes y ganaderos a los del campesino y habitante osornino que no tenía mayor influencia económica. Otro elemento que complementó el argumento del agro fue el fuerte discurso antimarxista de Sanhueza.

\section{EL RESPALDO DE RADIO SAGO A LA DICTADURA EN SUS PRIMEROS AÑOS}

En palabras de Carreño (2008), un 4 de septiembre de 1939, comerciantes, terratenientes y políticos locales de renombre iniciaron la emisora. La influencia de cada uno de estos miembros era significativa, desde dueños de fundos, exportadores de productos agrícolas, miembro de la SOFOFA (Sociedad de Fomento Fabril), comerciantes, dueños de bancos, directores de Club Osorno y autoridades públicas. Desde el primer minuto que su señal se unió al dial, representó y se fue construyendo como un dispositivo de comunicación al servicio del conglomerado agrícola. Como indica Santa Cruz (2016), «[...] hablar de comunicación era hablar de prácticas sociales» (p. 13).

El 11 de septiembre de 1973 la Junta Militar prohibió las transmisiones radiales. Recién el 19 del mismo mes, a través del bando 37, permite el 
trabajo periodístico de estos medios, pero bajo supervisión militar. La SAGO retornó al aire el 24 de octubre de 1973, bajo un irrestricto apoyo a los militares ¿Cuál fue su postura durante el primer año de gobierno? Se alinea totalmente a lo que podríamos denominar objetivos nacionales. El editorial del 2 de octubre estuvo marcado por un mensaje mercantilista, demagógico y con claras intenciones de crear y exacerbar un sentimiento antimarxista:

En esta hora que precisa decisiones y de rectificación para reestructurar en forma eficiente y patriótica [...] abandonar los procedimientos anacrónicos e insustanciales de una burocracia politizada y anarquizada por la propaganda marxista y crear una nueva mística que impulse la adopción de sistemas de trabajo eficientes (Sanhueza, 1973).

Una vez más, observábamos en las palabras de Sanhueza el miedo de la clase alta a cualquier institución que abogara por la diversificación, reestructuración, redistribución o democratización de las tierras, tildando de marxismo las visiones más progresistas. Como indica McLuhan (1997), «todos los medios son prolongaciones de alguna facultad humana, psíquica o física» (p. 26). En este caso, la SAGO utilizó su emisora para brindar un fuerte respaldo al nuevo sistema socioeconómico, impregnando su ideario de un marcado antimarxismo y apoyo al nuevo conglomerado al poder.

En ese momento fue recurrente el enaltecimiento a Adolfo Matthei (candidato del Movimiento Nacional Socialista en las parlamentarias de 1937) ¿Pero por qué no realzaron su postura a favor de una reforma agraria en el sur del país? Gallardo (2012) en su texto El ingeniero agrónomo Adolfo Matthei, cita al reconocido profesional: «Mientras no se vaya [...] al aumento de las pequeñas y medianas explotaciones, por medio de la subdivisión de la propiedad latifundista en Chile, la agricultura chilena jamás alcanzará el grado de progreso y la tranquilidad política [...]» (p. 73). En cambio, el común de los terratenientes hablaba de eficiencia laboral, pero nunca de las condiciones del hombre que realmente trabaja la tierra. «La relación de producción capitalista somete a los individuos al tratarlos únicamente como portadores de funciones económicas y solo eso» (Fernández, 2015, p. 60).

Es indudable que durante la UP existió desabastecimiento de los productos básicos. En el editorial del 29 de octubre de 1973, Sanhueza menciona «las colas» (filas), las JAP (Juntas de Abastecimiento y Control de Precios) y el mercado negro como elementos originarios del problema. En comparación al diario La Prensa, el editorial de la SAGO tergiversó y distorsionó la génesis del conflicto. Postulamos que SAGO tuvo una postura cómplice frente a las reales causas, no así La Prensa. El matutino hizo notar los mecanismos escogidos por los camioneros para desestabilizar la economía socialista, así lo evidencian variadas noticias publicadas en julio de 1974: «transportistas 
celebran primer aniversario de paro nacional», 21 de julio de 1974; «Hoy parte a Santiago caravana de camiones», 24 de julio de 1974; «Transportistas recuerdan su jaque al marxismo», 26 de julio de 1974.

Bajo esta misma lógica, Sanhueza (1973) creó una imagen limpia de Osorno, invisibilizando la proliferación de asentamientos periféricos:

Nuestra ciudad se caracteriza por [...] que le han transformado en forma acelerada en una hermosa urbe moderna de bellas construcciones y remozados barrios residenciales, haciendo desaparecer casi en su totalidad las poblaciones marginales y callampas ${ }^{4}$.

Aunque el mensaje de SAGO llegó al público en formato de audio, ayudó a reforzar lo que identificamos como un golpe estético (Errázuriz y Leiva, 2012), o para ser más precisos, la «estésis» propuesta por Mandoki (2006), un concepto de estética que reúne las expresiones artísticas y prácticas sociopolíticas, "la sensibilidad o condición de abertura, permeabilidad o porosidad del sujeto al contexto en que está inmerso [...] la estética aquí propuesta puede definirse como una estesiología filosófico-antropológica en cuanto aborda a los sentidos ya mediados por la cultura» (p. 50).

Además, es evidente que el apoyo ideológico de parte de los medios osorninos se constituyó por medio del terror, el recordatorio de la izquierda armada y su constante rearticulación, acusación que en la mayoría de los casos era infundada y exagerada. Según Augusto Pinochet, presidente de la Junta Militar de Gobierno y luego presidente de Chile, en un artículo publicado por diario La Prensa (1974) el 20 de abril: «Al otro lado de la frontera, catorce mil extremistas se estaban preparando, no tenemos temor. Se controlan las fronteras en forma exhaustiva $[\ldots] »^{5}$.

El miedo hacia los partidos de izquierda o disidentes a la Dictadura cívico-militar fue uno de los elementos más utilizados.

4 «A partir de los años 70 y con mayor fuerza a partir de la década de los 80, tanto las acciones surgidas producto de las políticas públicas de viviendas del Estado, con la política de relocalización socio espacial de la pobreza, básicamente acciones de erradicación de campamentos desde zonas centrales hacia otras más periféricas de la ciudad, en particular mediante el programa de mejoramiento de barrios P. M.B (Programa Mejoramiento de Barrios)». (Weibel y Angulo, 2013, p. 8)

5 De acuerdo con el reportaje Los montajes de la dictadura, el psicólogo Hernán Tuane Escaff emitía informes periódicos al Departamento de Relaciones Humanas y Conducta Social. En uno de los documentos fechados el 18 de noviembre de 1973 indicó que «es imprescindible reactivar los instantes angustiosos y trágicos previos al pronunciamiento militar» (citado en Luengo, 2015). También recomendó relacionar al marxismo con la violencia, escases, escándalo, angustia y peligro de muerte. 
Los elementos ideológicos que explican este posicionamiento se encuentran estrechamente vinculados a la Doctrina de la Seguridad Nacional [...], supone la existencia de un enemigo, el comunismo internacional, que socava las bases de sustentación de la convivencia nacional introduciendo el desorden social y político (Goicovic, 2012, p. 41).

De acuerdo con la conceptualización realizada por Martín (1973), la emisora se caracterizó por teñir sus informaciones de sensacionalismo, es decir: «Hechos políticos importantes son tratados como fuentes de miedo, excitación, alegría, alarma [...]» (p. 89).

Es primordial destacar que la postura de la SAGO era respaldada por el único medio escrito de la ciudad, el diario La Prensa (1975), quien intentó acercar a la Junta Militar al ideario portaliano, desligándolo de las características del imaginario fascista. "Nacionalismo portaliano», consignaba el editorial del 13 de octubre de 1975: «el nacionalismo portaliano no es de derecha ni izquierdista. Es solo de Chile y para Chile [...] Ese nacionalismo es lo que adelanta a Chile, rompiendo la historia».

Ya creados los enemigos internos y externos, la Radio SAGO con ayuda del matutino, se propuso brindar cierta identidad al proyecto de la Junta Militar. Montaron un ideario moral y político alrededor de Diego Portales, sin precisar exactamente a qué se referían, salvo pequeñas pinceladas cuando enarbolaban ideas como la libertad de mercado, orden estatal y recuperar «el espíritu» que caracterizaba a los chilenos antes de la llegada de la UP. La emisora y el diario incurrieron en constantes tergiversaciones y sesgos cuando se trató de enaltecer la figura de Portales. Nunca se le responsabilizó por atiborrar al país de mercaderes y rezagar la industrialización nacional. El análisis de la figura e ideario portaliano es meramente coyuntural, resaltaron el orden y la proliferación de negocios, haciendo a un lado las consecuencias a largo plazo.

El editorial del 13 de febrero de 1976 giró alrededor de los atributos patrios recuperados a partir del 11 de septiembre del 73:

El saneamiento ideológico que sobrevendrá cuando marche plena la institucionalidad [...] que persigue la exaltación espiritual del ciudadano, en su integridad y de su posibilidad de crearse los medios para su propia superación económica (Sanhueza, 1976).

La despolitización se instituyó como tema recurrente en el discurso de Sanhueza, al igual que la moral portaliana y segunda emancipación. La llamada libertad que vociferaba la emisora, estaba profundamente ligada al mero consumo. Como propone Sodré (1998, p. 51): 
Es posible observar que las exigencias de la economía de mercado imponen progresivamente un código cultural asimilador de todas las actividades humanas [...] donde los medios de comunicación masivos desempeñan un papel destacado [...] se debilita o se retrae la individualidad del sujeto, bajo la apariencia narcisista de que aumenta su autonomía, debido al mayor poder de elección de objetos de consumo.

Ahora, el discurso patriótico y de recuperación de estos atributos se mantuvo en el tiempo, e incluso construyeron nexos retrospectivos con los orígenes del país. La emisora realizó una analogía del 18 de septiembre de 1810 (primera Junta Nacional de Gobierno) y el 11 de septiembre de 1973 (Golpe de Estado):

Además del aniversario de la Independencia Nacional, esta efeméride se siente enfervorizada por la explosión patriótica del 11 de septiembre, tercer aniversario de la liberación nacional que nos devolvió la libertad y la dignidad soberana desquiciada por el gobierno marxista (Sanhueza, 1976).

Se asoció a los militares a una serie de libertades, no obstante, nunca fueron mencionadas, y cuando se hizo hincapié en la más notoria, la libertad de mercado, la SAGO llevó adelante una especie de contrapropaganda en desmedro del sistema económico, acusando una implementación ortodoxa del modelo. No obstante, como resulta evidente, el apoyo al ideario "éticomoral» se mantuvo como una importante caja de resonancia.

Ahora, bajo el lineamiento de lo moral, la libertad de tener una familia fue una de las ideas fuerza que levantó la casa radial. Bajo una campaña de desinformación política, creó la imagen de los partidos con tendencia marxista como destructores de esta estructura social:

Las esperanzas de un mundo más compasivo y generoso, concentra su pureza ritualista en el corazón de los hogares [...]. Y es ahí, hacia donde dirigen sus dardos envenenados aquellos que consideran a la integridad de la familia como la piedra granítica que sostiene a la civilización occidental (Sanhueza, 1976).

A eso se puede sumar el editorial del 4 de marzo de 1977, que centró su información en recalcar las supuestas hordas de jóvenes marxistas que asolaban las residencias hogareñas destruyendo ventanales. Fue una estrategia de desinformación profundamente inteligente. Identificaron con los partidos de izquierda uno de los principios de las corrientes anarquistas, como es el cuestionamiento a la jerarquía familiar. Siguiendo el análisis de Granel (2008), autores anarquistas como Malatesta incitaban su abolición, no así los partidos de izquierda. Estos últimos centraban sus análisis en la estructura socioeconómica de los países. 


\section{RADIO SAGO CRITICA AL GOBIERNO EN EL ÁMBITO ECONÓMICO}

En 1974 la actitud de la radioemisora no era de un apego total a la oficialidad, incluso, comenzó a lanzar duras críticas al manejo gubernamental en materia agronómica. De un irrestricto apoyo a la Dictadura y demonización de los grupos de izquierda, mantiene en su totalidad la segunda característica, matizando la primera de ellas. El 1 de febrero de 1974, el editorial recuerda lo que indican eran ciudadanos malagradecidos de la hecatombe social de la que fueron salvados, utilizando, al igual que La Prensa ${ }^{6}$, el Plan $\mathrm{Z}$ como arma para sembrar el terror:

A pesar de todos los sufrimientos y temores que arrojó la ola marxista, y de la masacre proyectada por la hora «Z», exista aún desmemoriados que pretendan olvidar tan reciente y tenebroso pasado [...] haciéndole un juego indirecto a aquellas células que en la clandestinidad actúan para tratar de mantener y acrecentar sus afanes revolucionarios (Sanhueza, 1974).

Ahora, es en el ámbito de la agricultura donde Sanhueza (1974) comienza a demostrar inconformidad con el desempeño estatal. Culpó al gobierno de no apoyar la actividad agrícola, pidiendo trato especial para la región de Los Lagos:

No se ha montado sobre un pie de estabilidad, la organización de una ayuda crediticia, amplia y complementada, siendo [...] la obra de una actividad burocratizada centralista que en cuanto a créditos, intereses y vencimientos carece de normas adaptadas a las características regionales.

La singularidad del mensaje de Sanhueza fue su reiterado llamado a un trato preferencial por parte del gobierno. Hacía parecer que la agricultura era una actividad que involucraba a todo habitante de Osorno, creando así un imaginario colectivo relacionado a esta actividad. Es homólogo a lo planteado por Benedict Anderson (2007) en Sociedad imaginada, cuando hace referencia a la creación ilusoria de las naciones, en este caso sería la pertenencia a una ciudad y actividad económica: «es imaginada porque aún los miembros de la nación más pequeña no obviando la actual desigualdad y explotación que puede prevalecer en cada una, la nación siempre se concibe como una camaradería profunda y horizontal» (p. 9).

$6 \quad$ Entre las noticias que mencionaban al Plan Z encontramos los siguientes títulos: «Amplían detalles de Plan Z», del 22 de noviembre de 1973; «Presuntos documentos del Plan Z», del 1 de diciembre de 1973 y «Radicales en el Plan Z», del 11 de diciembre de 1973. Es relevante destacar que dicho plan nunca existió, fue un invento de los uniformados en conjunto con los medios de comunicación. 
Bajo esta postura reafirmó su discrepancia con el manejo gubernamental del agro, apelando a la mala repartición de los recursos. Según sus impresiones, las ganancias de lo producido debían quedar en las arcas regionales:

En el detalle de las inversiones se puede observar un verdadero picadillo porque no se aprecia la existencia de una planificación que dé prioridad e importancia a las grandes soluciones capaces de provocar y acelerar el despegue socioeconómico de la provincia [...] al proveer de fondos provenientes de los recursos económicos de la provincia para programar la realización de proyectos nuevos y no como una ayuda a los ítems del presupuesto general de la nación (Sanhueza, 1974).

La crítica iba dirigida contra el centralismo administrativo aplicado por el régimen. A su juicio, las regiones quedaban como meros proveedores de ganancias, pero no veían materializada ninguna solución para sus rubros.

Ahora, resulta interesante constatar la conexión que hicieron entre la contaminación y la política, potenciando la imagen del gobierno a pesar de las discrepancias económicas mantenidas con el mismo. El editorial se mantuvo firme en su apoyo a los pilares ideológicos de la Dictadura: «La gran mayoría lucha por una vida más digna, libre de toda contaminación atmosférica y de toda influencia ideológica disociadora que prolifera en las poblaciones marginales» (Sanhueza, 1975).

Este es otro ejemplo de la «estésis» postulada por Mandoki (2006). Se asoció la ideología de izquierda con la pobreza, suciedad, desorden y proliferación de poblaciones marginales. Por esto mismo, la Junta Militar construyó su imagen en la vereda contraria. Aquello está plasmado en un documento de la Corporación de Estudios Nacionales publicado en 1983, en el que explicitaron su estrategia para vencer al marxismo: «Solo puede ser derrotada sobre la base de una vigorosa postura doctrinaria, estructurada por valores esencialmente opuestos a los suyos [...] nuestra concepción humanista de la vida, impregnada de sentido nacional y cristiano» Corporación de Estudios Nacionales (como citó en Donoso, 2019).

No obstante, el férreo respaldo en el ámbito «ético-ideológico» no se traspasaba al económico. Para finales de la década de los 80, Sanhueza (1979) expresaba incertidumbre frente al escenario económico, solicitaba que se escucharan a los diversos gremios presentes en la ciudad:

Desde los diversos sectores de la comunidad regional afloran inquietudes haciendo ver la necesidad que existe de dar una organización dinámica a las fuerzas vivas que representan el quehacer de la comunidad [...] para estudiar, planificar y proyectar los numerosos problemas. 
La SAGO, por medio de Sanhueza, daba la impresión de que el sistema económico iba a mostrar sus frutos en cualquier momento. Consideramos que el entusiasmo expresado por Sanhueza respondió al nulo conocimiento del experimento neoliberal. «Prácticamente, ningún país del mundo durante este periodo abrió su economía como lo hizo Chile. El país era un laboratorio —o más bien un "conejillo de indias" - de la ortodoxia teórica de la escuela económica de Chicago» (Gárate, 2012, p. 110). Y con la ayuda de los uniformados instalaron su itinerario sin un adversario ideológico que pudiera hacer frente a un proyecto que venía construyendo sus cimientos desde la década del 50 en las aulas de la Universidad Católica de Chile (Cardémartori, 2002)

\section{CREACIÓN DE UN DISCURSO REGIONALISTA}

Para mediados de la década del 70, la SAGO puso en circulación un discurso con un tono marcadamente regionalista. El editorial del 4 de julio de 1975 se puede encasillar como un ataque al libre mercado y la falta de industrialización regional:

Esta situación desfavorable de la producción agropecuaria del sur subsistirá mientras sea una región exportadora de materias primas, que entran a la competencia de los mercados, distorsionada por los sistemas de distribución, fletes caros e insuficientes (Sanhueza, 1975).

Si damos una mirada retrospectiva a la historia, era una crítica a la clase alta que estructuró la economía a partir de la Batalla de Lircay de 1830 . Gabriel Salazar (2018) responsabiliza a Diego Portales por permitir la excesiva instalación de mercaderes extranjeros (consignees), grupo que explotara excesivamente las materias primas, proyectando así un modelo económico desindustrializado y dependiente de las fluctuaciones internacionales.

Pues los consignes, al desembarcar, no buscaron aliarse con los artesanos o labradores, sino, de preferencia, con los comerciantes locales. No potenciaron, por eso, la capacidad productiva de la industria o la agricultura, sino, principalmente, las oportunidades de venta para las manufacturas [y otras especies] que ellos importaban (pp. 161-162) .

7 De acuerdo con André Gunder Frank (1968), la estructura de clases se comenzó a afianzar desde el arribo de los primeros conquistadores. Estos se apoderaron de las riquezas naturales y las explotaron a su favor, situación que se da hasta la actualidad, hecho que explicaría el subdesarrollo de los países latinoamericanos, y por ende 
¿Por qué recurrimos a la figura de Diego Portales? El 19 de junio de 1973 el editorial de la SAGO hacía alusión a la moral portaliana para sacar al país de su condición de crisis:

Las Fuerzas Armadas y del Orden y a los sectores de la actividad empresarial privada que con singular eficiencia estructuraron el código y la tradición de la moral portaliana [...] ¿Hasta cuándo? Hasta que aparezca como un mesías, la conciencia portaliana que arroje del templo de la honestidad a estos falsos mercaderes (Sanhueza, 1973).

En definitiva, Sanhueza presentó constantes manipulaciones e inconsecuencias en su discurso. En el tiempo de la UP, vanagloriaba y pedía un rescate de las huestes de mercaderes. Cuando el sistema estaba en todo su esplendor, el blanco de su reclamo variaba, ahora era el manejo estatal, no el sistema mercantil en sí mismo, ignorando completamente las consecuencias del destape económico que tanto añoraba.

Sanhueza posicionó a los ciudadanos osorninos como víctimas, no del gobierno, pero sí del centralismo, incitando a identificarse y construir su identidad regional por medio de la agricultura y las catástrofes que la aquejaban. Hizo masivas las problemáticas de una actividad que resulta significativa para la región, pero no por ello era el foco de las problemáticas sociales que asolaban a la urbe sureña. Ahora, si observamos el mensaje de Sanhueza desde la perspectiva de Marx (1989, p. 7), era relevante interiorizar la problemática agrícola al común de los ciudadanos para que actuaran acorde a la élite terrateniente:

El modo de producción de la vida material condiciona el proceso de la vida social, política y espiritual en general. No es la conciencia del hombre la que determina su ser, sino por el contrario, el ser social es lo que determina su conciencia.

El editorial de radio SAGO se apoderó y moldeó a la opinión ciudadana, transmitiendo lo que a su juicio era una postura por todos compartida. Sin duda alguna fue un diálogo asimétrico con la sociedad osornina, instalando forzosamente la opinión de los agricultores como verdad única con relación a la economía regional.

En octubre de 1975, ya transcurridos poco más de dos años de Dictadura cívico-militar, Sanhueza (1975) redactó un editorial cargado de ira, no contra el gobierno, pero atacó al que podríamos denominar el padre del modelo

la subyugación a las naciones que se construyeron como potencia, gracias a la venta de productos manufacturados confeccionados con la materia prima extraída desde estas tierras. 
económico neoliberal, Estados Unidos. Responsabilizó al país norteamericano de intervenir la economía nacional:

Estas desviaciones del bien social, provocadas por los imperialismos económicos e ideológicos, que se han enseñoreado de los medios de producción y de los beneficios materiales que de ellos obtienen [...] el egoísmo de los soberanos de la Quinta Avenida de Nueva York siguió estrujando a los países subdesarrollados, extrayéndoles sus materias primas minerales, y empobreciéndolas hasta convertirlas en hermanas pobres a las que había que socorrer.

¿Qué pretendió Sanhueza con estas palabras cargadas de acusaciones? Percibimos gran decepción sobre el modelo económico que el mismo solicitaba: libertad de precios, libertad de comercio, que el Estado no se entrometiera en asuntos económicos, entre otros.

Fue constante la figura del ciudadano osornino como una víctima del sistema, lo que ayudó a afianzar la identidad regional. Creó enemigos nacionales, la centralización del sistema administrativo, falta de trato preferencial hacia la actividad agropecuaria, la izquierda, entre otros. Además, la SAGO ideó otro elemento de inseguridad, pero esta vez con un enemigo de peso mayor, el padre del sistema económico neoliberal, Estados Unidos. A primera vista resulta extraño toparnos con esta fuerte crítica hacia la nación que arrebató el poder al conglomerado socialista. No obstante, Sanhueza instauró un combativo discurso de industrialización para la región de Los Lagos, según sus palabras, era el camino para la anhelada independencia económica.

La emisora pareció olvidar que la Dictadura cívico-militar dio los primeros pasos hacia lo que sería uno de los experimentos sociales pioneros en el mundo, el neoliberalismo en todo su esplendor. Una investigación llevada adelante a principio de los 80 arrojó cifras críticas sobre la situación en que se encontraba la industria nacional. Todos los ítems consultados, que iban desde insumos elementales como trigo, maíz y azúcar, marcaban un alza en su nivel de importaciones (French, 1980).

La intromisión del capital extranjero en la banca, comercio e industria hizo que la manufactura chilena fuera quedando en el olvido, y como bien puntualizaba Sanhueza, Chile era (y es) un mero productor de materias primas.

¿Por qué Sanhueza se sorprendió del vuelco que tuvo la economía? Recapitulando, he allí la inconsistencia de su argumentación. Hemos realizado un análisis sobre su evolución discursiva, y transitó de una angustiosa solicitud de liberalización del mercado, citando al padre de los mercaderes, Diego Portales, a un trato especial cuando el neoliberalismo dejaba sus primeros estragos. Alfonso Sanhueza no logró entender el funcionamiento y consecuencias que acarreó la implementación de un libre mercado en una sociedad como la chilena. 
Los intereses de un individuo tal como el de la teoría de mercado [...] se definen como esencialmente independientes o desvinculados de los de otra gente, y sus transacciones con el mundo consisten fundamentalmente de muchos intentos de obtener satisfacción de esos intereses privados (Plumwood, 2003, p. 254).

A pesar de todo lo antes relatado, a mediados de la década de los 70, Sanhueza (1977) aún asentaba su convicción en la libertad de mercado y la superación personal por sobre las aspiraciones colectivas. No obstante, en octubre de 1977 comienza a tomar fuerza uno de los altercados más recordados en la historia del agro nacional, el llamado «Conflicto de la Leche». El Secretario General de la SAGO acusaba malas prácticas de parte de las empresas lecheras:

Una grave amenaza se cierne sobre la producción de leche [...] una competencia dumping que se ha dejado caer sobre la zona franca alimenticia del norte [...], el precio de relación es inferior al que pagan las plantas lecheras regionales a sus proveedores y en consecuencia los capitales foráneos están aplicando el tan conocido procedimiento de la importación de productos similares para ejercer un dumping sobre la producción nacional.

Según la conceptualización de Crespo (1997), el dumping es una práctica donde se vende por debajo del precio referencial e incluso de producción del producto, con la finalidad de monopolizar el mercado. Considerando aquello, es importante aclarar que los productores de leche agrupados en la Confederación de Productores Agrícola (CPA), además de ser representados por la Sociedad Nacional de Agricultura (SNA), eran un ínfimo y selecto grupo de capitalistas agrícolas.

En resumidas cuentas, el 23 de julio de 1977, el gobierno decidió bajar el arancel por tonelada de leche de 300 a 130 dólares, argumentando la fuerte alza a nivel mundial. Evidentemente, los agricultores del sur alzaron inmediatamente la voz y tomaron acciones. "El presidente de la CPA anuncia la inminente quiebra de la industria lechera nacional y señala que ya han sostenido una reunión de urgencia en Osorno con dirigentes productores de la zona»(Gómez, 1982, p. 177).

El conflicto se transformó en un juego de poder entre los productores de leche y el gobierno. Como era de esperarse, la SAGO puso sus micrófonos a disposición de las demandas agrícolas. El 27 de julio el editorial, nuevamente, fue dedicado a denunciar el dumping generado por el libre mercado:

Los precios, y aun cuando han sido liberados del régimen estatal de los precios políticos, no obstante, la convalecencia es casi peor que la enfermedad [...] ¿Y qué sucede con la leche? Se ha abierto la llave de la importación de leche en 
polvo y posiblemente mantequilla [...], es lógico suponer que van en «dumping» con la producción nacional (Sanhueza, 1977).

Si observamos cuidadosamente el discurso de Sanhueza nos podríamos preguntar ¿dónde está representado el ciudadano medio que no es productor, pero si consumidor de lácteos? Simplemente no existe, el único actor reflejado fue el gran agricultor, el productor acaudalado con gran número de ganado.

La ofensiva de la SAGO se mantuvo activa durante todo el proceso de coletazos entre agricultores y gobierno. El 1 de agosto Sanhueza (1977) direccionó su discurso hacia la demagogia chovinista:

Es nocivo para el interés nacional y para el proceso normal de la producción, suplir a destajo y sin consideración alguna, el deber superior de defender la producción interna, por aquella afición morbosa de internar sustitutos, pagando en divisas tan valiosas, los alimentos que se producen en el país, aunque cuesten más que los importados, pero en todo caso, son obtenidos del suelo chileno y mediante el esfuerzo de los labradores.

La argumentación técnica fue nula. Incluso reconoció que la importación de productos resultaba más conveniente. Repetidamente expuso los intereses de los grandes agricultores como si estos se tradujeran en beneficios inmediatos para la población en general. Es a través de estos mecanismos que la SAGO intentó construir una identidad regional en base a la agricultura.

El discurso de Sanhueza (1977) dio un giro en sus conceptos más utilizados. Radio SAGO y el diario La Prensa se referían a las corrientes de izquierda como doctrinas foráneas, atribuyendo al concepto una serie de características negativas. En el editorial del 10 de agosto de 1977 la SAGO utilizó el mismo adjetivo para referirse al sistema económico que había implementado la Dictadura cívico-militar:

La producción de leche es sometida a un tratamiento inspirado en doctrinas económicas foráneas, que pretende regularizar el valor de la leche nacional con la producida en los países exportadores de dicho producto y que la expenden bonificada, porque lo normal y lógico sería consumir lo que es chileno, aun cuando fuera más caro.

La periodista canadiense Naomi Klein (2008) sostiene que el neoliberalismo en todo su esplendor, como se impuso en Chile, solo pudo ser implantado a través de la fuerza, durante un Golpe de Estado, un shock. Vale decir, el discurso de Sanhueza era un reflejo del duro traspié que significó la extrema apertura económica para el agro nacional. 
En un primer momento, solicitaban la liberación de precios y retiro del Estado en materia económica, dejando sus micrófonos al servicio del afianzamiento del nuevo sistema. El irrestricto apoyo se iría debilitando con el paso del tiempo hasta adquirir una actitud crítica frente a las medidas gubernamentales. No obstante, se configuraron como fervientes representantes del itinerario "ético-valórico»: enaltecimiento de símbolos patrios, familia, FF.AA., consignas nacionalistas, depuración de partidos y movimientos de izquierda, entre otros. Más que apoyar el ideario nacionalista, la SAGO se encargó de crear el imaginario de que el nuevo gobierno favorecería la anhelada industrialización regional.

¿En qué terminó la pugna entre productores de leche y Estado? El arancel se estableció en US $\$ 248$ dólares, y consignaron la compra obligatoria de la leche en polvo a productores nacionales. Únicamente después de haber cubierto la cuota nacional podrían importar. Finalmente, el gobierno cedió ante la demanda de los productores ¿Cuáles fueron las causas que posibilitaron la victoria de los sureños? Este grupo se caracterizaba por su homogeneidad: se concentraban mayoritariamente en la Provincial de Llanquihue, lo que les otorgaba una mayor capacidad de presión, tenencia mediana a grande de tierras (50 a 200 hectáreas), y gran parte de los productores eran descendiente de colonos europeos (homogeneidad cultural) (Gómez, 1982).

Si analizamos la actuación de la SAGO desde la perspectiva de Foucault (1977), este conceptualiza al dispositivo como "un conjunto resultante heterogéneo que compone los discursos, las instituciones [...], las decisiones reglamentarias, las leyes» (p. 229). Es posible decir que los agricultores utilizaron la emisora como un dispositivo a su favor, la acción propagandística tuvo características esencialmente estratégicas, manipularon las relaciones de fuerza a su favor, dislocaron la resistencia gubernamental a mantener el precio de la tonelada de leche. Es un caso bastante puntual, pero ayuda a dimensionar el poder que los agricultores de la décima región ostentaban en aquellos años.

Es cierto que es un caso como tantos otros, aunque debido a sus características (extrema apertura a capitales foráneos), se puede diagnosticar un problema estructural.

¿Cómo se podría catalogar el episodio?: ¿cómo una derrota gubernamental o del neoliberalismo frente a los terratenientes? Los productores de leche fueron los únicos beneficiados, si bien no es un escándalo de proporciones catastróficas, la imagen del gobierno se vio deteriorada, la entidad pecuaria ganó el juego de poder, los intereses privados primaron sobre los del bien común. 
El sistema no fue modificado estructuralmente. El Estado adquirió un compromiso con los productores, pero el resto de la economía continuó su frenética apertura a las divisas extranjeras. De hecho, Radio SAGO, a pesar de atacar la falta de ayuda para el agro, continuaba vanagloriando la libertad de consumo. De acuerdo con Sodré (1998), en los países de capitalismo periférico, los medios cumplen esa función: «[...] haciendo creer a todos que ése es el camino para una verdadera democracia social» (p. 82).

Por otra parte, el constante sentimiento regionalista que expresaba Sanhueza se entremezclaba con las ansias de desprenderse de las ayudas económicas estatales. El Secretario General de la SAGO visualizaba en el proyecto de regionalización una oportunidad para la anhelada independencia económica: «El nuevo proceso de regionalización del país [...] traerá a las diversas regiones el estímulo necesario para poner en marcha sus propios recursos, que las lleve al desarrollo agroindustrial» (Sanhueza, 1977).

Las discrepancias que Sanhueza expresaba del modelo económico tuvieron relación con la apertura de las barreras nacionales al capital foráneo. Pese a aquello, el locutor de la SAGO lo proyectaba como un neoliberalismo a nivel nacional, dejando el mercado libre de restricciones dentro de nuestras fronteras y cerrando paulatinamente las oportunidades de inversión al extranjero. El tiempo dejó en evidencia que su perspectiva tenía muchas diferencias con los teóricos de Chicago.

\section{CONCLUSIONES}

Durante los primeros años de gobierno de la Junta Militar, la SAGO desplegó un discurso de respaldo al nuevo conglomerado al poder. El concepto de patria y todos sus elementos asociados se conjugaron en la consigna de aunar el país bajo el nuevo proyecto político. Sin lugar a duda, recurrieron a la figura del estadista Diego Portales para personificar los objetivos de su gobierno, aunque, como ya mencionamos, sin profundizar en los reales alcances de su política económica. El proyecto país (al menos en el papel) emanaba un futuro promisorio (industrialización, independencia económica, trabajo y economía estable, entre otros). Al mismo tiempo se encargaban de vilipendiar lo realizado por la UP y las tendencias de izquierda o marxistas, instaurando el terror constante a la rearticulación de estos partidos.

Otra de las aristas en el discurso de Sanhueza fueron sus constantes críticas al manejo agrario, aunque, manteniendo su apoyo al ideario «ideológicomoral». Ahora, a pesar de vociferar un acérrimo apoyo al libre mercado, los 
puntos señalados por la SAGO mostraban las serias fisuras del plan económico gubernamental. La crítica a nivel global eran la falta de ayuda: centralismo y burocratización excesiva. En resumidas cuentas, a pesar de pertenecer al mismo sector político, la SAGO divulgó la imagen de un gobierno que improvisaba en materia agraria y cedía ante las demandas de la economía internacional. Sin lugar a duda la SAGO resultó ser un gran aliado de la Dictadura, pero a la vez un arma de doble filo.

Además del apoyo a la Dictadura y crítica en materia económica, se suma una tercera idea, el despliegue de un marcado discurso regionalista. A diferencia del punto dos, en este apartado hacen notar con más fuerza lo que ellos consideraban una desventaja regional para competir de igual a igual con los productores extranjeros. Acusaron un constante retraso en la implementación de medidas gubernamentales que se tradujeran en la esperada industrialización. Ahora, es durante el Conflicto de la Leche cuando hacen notar que, a pesar de estar alineado con los puntos centrales del gobierno, la SAGO prefirió comprometer su apoyo a los productores locales, aunque eso significara dañar la imagen de la Dictadura.

\section{REFERENCIAS}

Anderson, B. (2007). Comunidad imaginada. Ciudad de México, México: F.C.E.

Canales, M. (2006). El saber cualitativo. En Canales, M (ed.). Metodología de investigación. Introducción a los oficios. (pp. 19-24). Santiago, Chile: LOM.

Cardémartori, J. (2002). Neoliberalismo y globalización en Chile. En A. Boron y J. Gambina, (ed.). La globalización económico-financiera. Su impacto en América Latina. (pp. 371-376). Buenos Aires, Argentina: CLACSO.

Carreño, L. (2008). La Sociedad Agrícola y Ganadera de Osorno (SAGO) y su aporte al desarrollo de la comunidad regional. Espacio Regional, 5(1), 35-48.

Comisión Nacional de Verdad y Reconciliación (1990). Informe de la Comisión Nacional de la Verdad y Reconciliación. Disponible en http://bibliotecadigital.indh.cl/ handle/1234 56789/170

Comisión Nacional sobre Prisión y Tortura (2011). Informe Comisión Nacional sobre Prisión Política y Tortura. Disponible en http://bibliotecadigital.indh.cl/handle/1234567 89/455

Crespo, J. (1997). Dumping, antidumping y fundamentos del antidumping. Revista Venezolana de Análisis de Coyuntura, 3(2), 1-20.

Donoso, K. (2019). Cultura y dictadura. Censuras, proyectos e institucionalidad cultural en Chile, 1973-1989. Santiago, Chile: Ediciones Universidad Alberto Hurtado.

Errázuriz, L., y Leiva, G. (2012). El golpe estético. Dictadura militar en Chile 19731989. Santiago, Chile: Ocho Libros. 
Fernández, C. (2015). El marxismo hoy. La herencia de Gramsci y Althusser. Barcelona, España: Batiscafo S.L., Bonalletra S.L.

Ffrench, R. (1980). Liberalización de las importaciones: la experiencia chilena en 1973- 79. Colección Estudios CIEPLAN, 2 (4), 39-78.

Foucault, M. (1977). Dits et écrits (vol III). París, Francia: Gallimard.

Gallardo, E. (2012). Ingeniero agrónomo Dr. Adolfo Matthei y su rol en el desarrollo de la agricultura en Chile y Osorno (1925-1939). Osorno, Chile: ULA.

Gárate, M. (2012). El nuevo estatus del economista y el papel de los think tanks en Chile: el caso de Cieplan. En T. Ariztía (ed.), Produciendo lo social (pp. 101-132). Santiago, Chile: Ediciones UDP.

Goicovic, I. (2012). Movimiento de izquierda revolucionaria. Santiago, Chile: Escaparate.

Gómez, S. (1982). Organizaciones patronales y gobierno militar. Análisis del conflicto de la leche de 1977 («Que se coman las vacas»). En Gómez, S (ed.), Instituciones $y$ procesos agrarios en Chile. (pp. 111-126). Santiago, Chile: FLACSO.

Granel, H. (2008). Anarquismo y sexualidad. Revista de Estudios Libertarios Germinal, 1 (5), 65-84.

Gunder Frank, A. (1968). Latinoamérica: subdesarrollo capitalista o revolución socialista. Pensamiento Crítico, 1(13), 3-41.

Joselyn-Holt, A. (1998). El peso de la noche. Nuestra frágil fortaleza histórica. Santiago, Chile: Editorial Planeta Chile.

Klein, N. (2008). La doctrina del shock. El auge del capitalismo del desastre. Buenos Aires, Argentina: Paidós.

Luengo, A (Director). (2015). Los montajes de la dictadura [Programa de televisión]. En Abner Machuca (Productor). Informe Especial. Santiago, Chile: TVN.

Mandoki, K. (2006). Estética cotidiana y juegos de la cultura: Prosaica I. Ciudad de México, México: Siglo XXI Editores.

Martín, G. (1973). Discrimination and Popular Culture. Londres, Reino Unido: Penguin Books.

Marx, K. (1989). Contribución a la crítica de la economía política. Moscú, Rusia: Editorial Progreso.

Pérez, G. (2001). Investigación cualitativa. Retos e interrogantes. Métodos. Madrid, España: Editorial La Muralla.

Plumwood, V. (2003). Naturaleza, yo y género: feminismo, filosofía del medioambiente y crítica del racionalismo. En K. Warren (coord.). Filosofía ecofeministas (pp. 253-288). Barcelona, España: Icaria Editorial.

S.N. (1973, 1 de diciembre). Presuntos documentos de Plan Z. La Prensa, p. 2.

S.N. (1973, 21 de noviembre). Amplían detalles de Plan Z. La Prensa, p. 9.

S.N. (1974, 26 de julio). Transportistas recuerdan su jaque al marxismo. La Prensa, p. 9.

S.N. (1974, 20 de abril). 14 mil extremistas pretenden invadir nuestro territorio. $\mathrm{La}$ Prensa, p. 7.

S.N. (1974, 21 de julio). Transportistas celebran primer aniversario de paro nacional, La Prensa, p. 7.

S.N. (1974, 24 de julio). Hoy parte a Santiago caravana de camiones. La Prensa, p. 7.

S.N. (1973, 11 de diciembre). Radicales en el Plan Z. La Prensa. p. 4. 
Salazar, G. (2018). Mercaderes, empresarios y capitalistas. Santiago, Chile: Debate.

Salgado, A. (2007). Investigación cualitativa: diseños, evaluación del rigor metodológico y retos. Liberabit, 13(13), 71-78.

Sanhueza, A. (Productor). (1973, 11 de abril). Editorial [Transmisión radial]. Osorno: Radio SAGO.

Sanhueza, A. (Productor). (1973, 14 de octubre). Editorial [Transmisión radial]. Osorno: Radio SAGO.

Sanhueza, A. (Productor). (1973, 19 de junio). Editorial [Transmisión radial]. Osorno: Radio SAGO.

Sanhueza, A. (Productor). (1973, 20 de junio). Editorial [Transmisión radial]. Osorno: Radio SAGO.

Sanhueza, A. (Productor). (1973, 20 de octubre). Editorial [Transmisión radial]. Osorno: Radio SAGO.

Sanhueza, A. (Productor). (1973, 26 de septiembre). Editorial [Transmisión radial]. Osorno: Radio SAGO.

Sanhueza, A. (Productor). (1973, 29 de octubre). Editorial [Transmisión radial]. Osorno: Radio SAGO.

Sanhueza, A. (Productor). (1974, 1 de febrero). Editorial [Transmisión radial]. Osorno: Radio SAGO.

Sanhueza, A. (Productor). (1974, 6 de marzo). Editorial [Transmisión radial]. Osorno: Radio SAGO.

Sanhueza, A. (Productor). (1974, 9 de febrero). Editorial [Transmisión radial]. Osorno: Radio SAGO.

Sanhueza, A. (Productor). (1975, 11 de octubre). Editorial [Transmisión radial]. Osorno: Radio SAGO.

Sanhueza, A. (Productor). (1975, 25 de octubre). Editorial [Transmisión radial]. Osorno: Radio SAGO.

Sanhueza, A. (Productor). (1975, 4 de julio). Editorial [Transmisión radial]. Osorno: Radio SAGO.

Sanhueza, A. (Productor). (1976, 13 de febrero). Editorial [Transmisión radial]. Osorno: Radio SAGO.

Sanhueza, A. (Productor). (1976, 16 de septiembre). Editorial [Transmisión radial]. Osorno: Radio SAGO.

Sanhueza, A. (Productor). (1976, 25 de octubre). Editorial [Transmisión radial]. Osorno: Radio SAGO.

Sanhueza, A. (Productor). (1976, 27 de diciembre). Editorial [Transmisión radial]. Osorno: Radio SAGO.

Sanhueza, A. (Productor). (1977, 01de agosto). Editorial [Transmisión radial]. Osorno: Radio SAGO.

Sanhueza, A. (Productor). (1977, 08 de junio). Editorial [Transmisión radial]. Osorno: Radio SAGO.

Sanhueza, A. (Productor). (1977, 10 de agosto). Editorial [Transmisión radial]. Osorno: Radio SAGO.

Sanhueza, A. (Productor). (1977, 27 de julio). Editorial [Transmisión radial]. Osorno: Radio SAGO. 
Sanhueza, A. (Productor). (1979, 11 de enero). Editorial [Transmisión radial]. Osorno: Radio SAGO.

Santa Cruz, E. (2016). Prólogo a la segunda edición. En G. Sunkel (coord.). Razón y pasión en la prensa popular. Un estudio sobre la cultura popular de masas y cultura política. Santiago, Chile: Ediciones y publicaciones El Buen Aire S.A.

Sodré, M. (1998). Reinventando la cultura. Barcelona, España: Gedisa.

Sorensen, K. (2015). Los medios, la memoria y los derechos humanos en Chile. Santiago, Chile: RIL.

Weibel, H., y Angulo, A. (2013). Transversalidad continental. Osorno, espacio urbano en tensión. Estudios Trasandinos, 18(1), 121-130. 